\title{
Monolithic Broadband Gilbert Micromixer With an Integrated Marchand Balun Using Standard Silicon IC Process
}

\author{
Sheng-Che Tseng, Student Member, IEEE, Chinchun Meng, Member, IEEE, Chia-Hung Chang, Chih-Kai Wu, and \\ Guo-Wei Huang
}

\begin{abstract}
A single-ended wideband downconversion Gilbert micromixer is demonstrated in this paper using $0.35-\mu \mathrm{m} \mathrm{SiGe}$ BiCMOS technology. A transimpedance amplifier with resistive feedback is utilized in the IF stage while a broadband Marchand balun is employed to generate wideband differential local oscillator signals. The planar Marchand balun topology employed in this paper can generate truly balanced signals even in the presence of the lossy low-resistivity $(\sim 10 \Omega \cdot \mathrm{cm})$ silicon substrate. A systematic approach to measure the frequency response of each individual stage in a Gilbert mixer is developed in this paper. This single-ended wideband mixer has the conversion gain of $15 \mathrm{~dB}$, IP $_{1 \mathrm{~dB}}$ of $-19 \mathrm{dBm}, \mathrm{IIP}_{3}$ of $-7 \mathrm{dBm}$, and the noise figure of $13 \mathrm{~dB}$. The mixer works from 3.5 to $14.5 \mathrm{GHz}$.
\end{abstract}

Index Terms-Downconverter, Marchand balun, micromixer, SiGe BiCMOS, silicon substrate, transimpedance amplifier (TIA), wideband.

\section{INTRODUCTION}

$\mathbf{T}$ THE ERA of the wireless applications with high data-rate transmission and multiple functions is coming, e.g., the IEEE $802.11 \mathrm{a} / \mathrm{b} / \mathrm{g}$ combo system [1], ultra-wideband (UWB) system [2], and WiMAX system [3]. The range of carrier frequencies and their bandwidth constantly increase. The obligation of the complicated data processing belongs to the baseband design, while the RF integrated circuit (IC) design takes responsibility for the wide range frequency and broad bandwidth operation. Nevertheless, the design of the high-frequency and wideband RF circuits is a big challenge in the overall solution implementation. For an active mixer, the transistors have natural instinct to perform wide range and broad bandwidth frequency translation. Due to the input/output matching networks, narrowband passive components, and loading effects, the mixer's wideband ability is restricted.

Manuscript received March 31, 2006; revised July 25, 2006. This work was supported by the National Science Council of Taiwan, R.O.C., under Contract NSC 95-2752-E-009-001-PAE and Contract NSC 95-2221-E-009-043-MY3, by the Ministry of Economic Affairs of Taiwan under Contract 94-EC-17-A-05-S1020, by the Ministry of Education Aim for Top University Program under Contract 95W803, and by the National Chip Implementation Center.

S.-C. Tseng, C. Meng, and C.-H. Chang are with the Department of Communication Engineering, National Chiao Tung University, Hsinchu 300, Taiwan, R.O.C. (e-mail: ccmeng@mail.nctu.edu.tw).

C.-K. Wu was with the Department of Communication Engineering, National Chiao Tung University, Hsinchu 300, Taiwan, R.O.C. He is now with Agilent Technologies, Tao-Yuan 324, Taiwan, R.O.C.

G.-W. Huang is with National Nano Device Laboratories, Hsinchu, 300 Taiwan, R.O.C.

Digital Object Identifier 10.1109/TMTT.2006.884690
For the wideband circuit design, the wideband matching of the input/output ports is a significant issue. The common implemented active mixer is a Gilbert mixer using the emitter-coupled differential input stage. Owing to the high input impedance of the common-emitter-configured transistors, the reactive or resistive matching is needed at the input port. For the reactive matching, the matching bandwidth relates to the orders of the passive matching network. Increasing the order of the matching network can expand the operation bandwidth, but also takes more area. Although the resistive matching can perform wideband matching, it also introduces loss. The variant of the Gilbert mixer, the so-called micromixer, which is defined as a microwave mixer in [4], has the properties of the wideband input matching and single-ended input. Those properties facilitate the realization of the wideband and single-ended mixer. In this paper, the input stage of the mixer is made up of the micromixer.

For the balanced mixers, the Gilbert switch quad demands differential local oscillator (LO) signals. It is cumbersome to use an off-chip balun for the wideband balanced LO signal generation because the differential signals experience the different delay paths on the circuit board, especially at high frequencies. Hence, a single-to-differential LO balun is integrated in the IC process to form a single-ended mixer. Since it is difficult to achieve truly differential signals with equal magnitude and opposite phase by an active balun in addition to more power consumption at high frequencies, a passive balun is taken into consideration. The Marchand balun is a very wideband passive balun and is popularly used for broadband applications such as a double-balanced diode mixer [5] and a frequency doubler [6]. However, most Marchand baluns are realized on a semi-insulating or high-resistivity substrate. The proper Marchand balun topology suitable for a standard silicon IC process is identified in this paper to maintain the truly balanced signals regardless of the substrate loss.

High impedance resistors or active pMOS loads are usually employed to obtain high conversion gain. In addition, the pMOS current mirror is used to effectively combine the differential IF output current signals of the mixer and establish a singleended output. However, the high impedance causes a low-frequency pole at the output stage, which slows down the IF response. The transimpedance amplifier (TIA) with resistive feedback is, hence, utilized at the output stage to reduce the output impedance and extend the bandwidth in this paper [7], [8].

A single-ended wideband Gilbert downconverter is fabricated in the $0.35-\mu \mathrm{m}$ SiGe BiCMOS technology and demonstrated in 


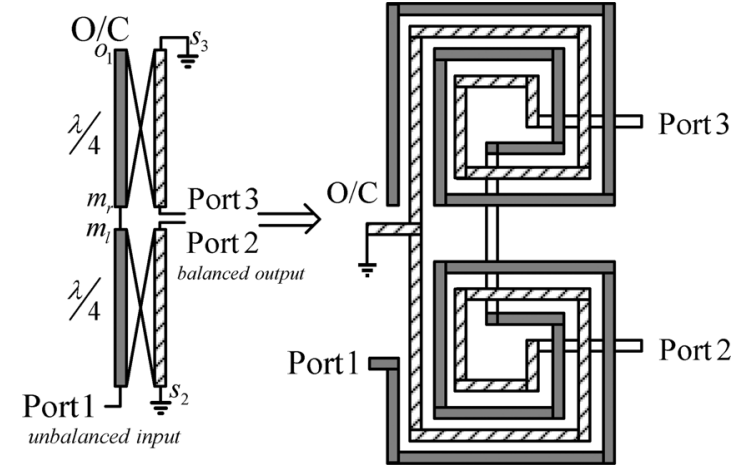

Fig. 1. Planar Marchand balun with $\lambda / 4$ coupled lines.

this paper. It is composed of a micromixer, an integrated LO Marchand balun, and a TIA output amplifier. In this paper, a technique to measure the RF, LO, and IF stages of a Gilbert mixer is developed. This mixer has $15-\mathrm{dB}$ conversion gain, $13-\mathrm{dB}$ noise figure, and $400-\mathrm{MHz}$ IF bandwidth and works from 3.5 to $14.5 \mathrm{GHz}$.

The Marchand balun design concept and the measured results of a monolithic planar Marchand balun are represented in Section II, and Section III depicts the entire circuits of the micromixer with an integrated Marchand balun. Section IV then shows the experimented results, including the performances of an individual micromixer and an overall micromixer with an integrated Marchand balun. Finally, Section V gives a conclusion with a brief summary of the mixer's performances.

\section{ANALYSIS AND IMPLEMENTATION OF THE PLANAR MARCHAND BALUN ON SILICON IC PROCESS}

\section{A. Analysis}

The Marchand balun, a very broadband passive balun, was proposed in 1944 and has one unbalanced input and two balanced outputs [9]. The compensated Marchand balun can perform impedance transformation from the balanced port to the unbalanced port. The load at the balanced port is shunted with a quarter-wavelength short stub and in series with a quarter-wavelength open stub [10], [11]. Nevertheless, this type is not easily realized in the IC process, especially in the silicon IC process, and thus is not commonly used in ICs.

The planar Marchand balun is composed of two back-to-back quarter-wavelength coupled lines, as shown in Fig. 1. Each coupled line has four ports-input, direct, coupled, and isolated ports. Two coupled ports of coupled lines are connected with short ends; the direct ports are tied together. One of the input ports is connected with an open end and the other is the unbalanced input of the Marchand balun, while the balanced outputs of the Marchand balun are from the isolation ports. This configuration is the most popular one, and other topologies of the Marchand balun had been developed in [12].

The transmission and reflection properties of the Marchand balun can be analyzed easily by the properties of the coupler

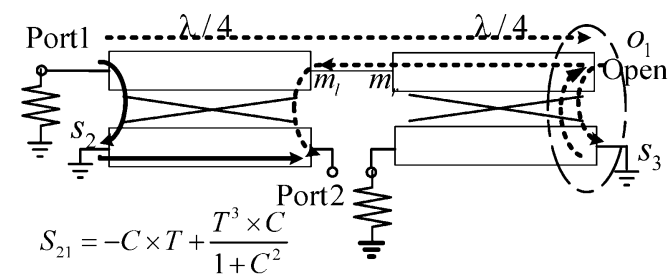

(a)

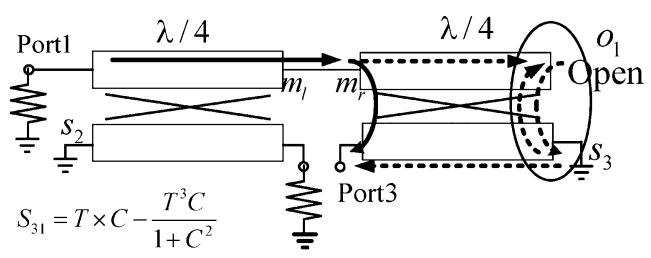

(b)

Fig. 2. $S$-parameter derivation of the planar Marchand balun with $\lambda / 4$ coupled lines. (a) $S_{21}$. (b) $S_{31}$.

and open and short terminals [13]. The quarter-wavelength coupled line has the scattering parameters for the coupled and transmitted ports $C$ and $T$, which are derived in Appendix. The relation between $C$ and $T$ of the coupled line is written as

$$
\begin{cases}|C|^{2}+|T|^{2}=1, & \text { no loss } \\ |C|^{2}+|T|^{2}<1, & \text { with loss. }\end{cases}
$$

The short terminal results in an antiphase total reflection, whereas the open terminal causes an in-phase total reflection. When a signal inputs at port 1, one part of the input signal, the solid line signal, shown in Fig. 2(a), couples to the short terminal, then reflects totally in an antiphase fashion, and finally transmits to port 2 . This causes the $C \times(-1) \times T$ voltage wave transmitting to port 2 . The other part, the dotted line signal, is analyzed more complicatedly, as shown in the following steps.

Step 1) The dotted line signal transmits to the open terminal $o_{1}$ and reflects totally.

Step 2) Some reflected power directly transmits to the middle and then couples to port 2 ; the rest power couples to the short terminal $s_{3}$.

Step 3) A proportion of power reflected from the short terminal $s_{3}$, couples to the open terminal, $o_{1}$, and then reflects totally. Finally, the reflected signal progresses repeatedly from Step 2).

Consequently, the transmission coefficient form ports 1 to 2 caused by the dotted line signal is $T^{3} C /\left(1+C^{2}\right)$. Therefore, the total transmission coefficient from ports 1 to 2 is

$$
S_{21}=-C \times T+\frac{T^{3} \times C}{1+C^{2}} .
$$

With the same analysis approach, the total transmission coefficient form ports 1 to 3 is

$$
S_{31}=C \times T-\frac{T^{3} \times C}{1+C^{2}}
$$

as shown in Fig. 2(b). Based on the calculations of $S_{21}$ and $S_{31}$, this balun performs single-to-differential conversion perfectly, regardless of the silicon substrate loss and metal loss, thanks 


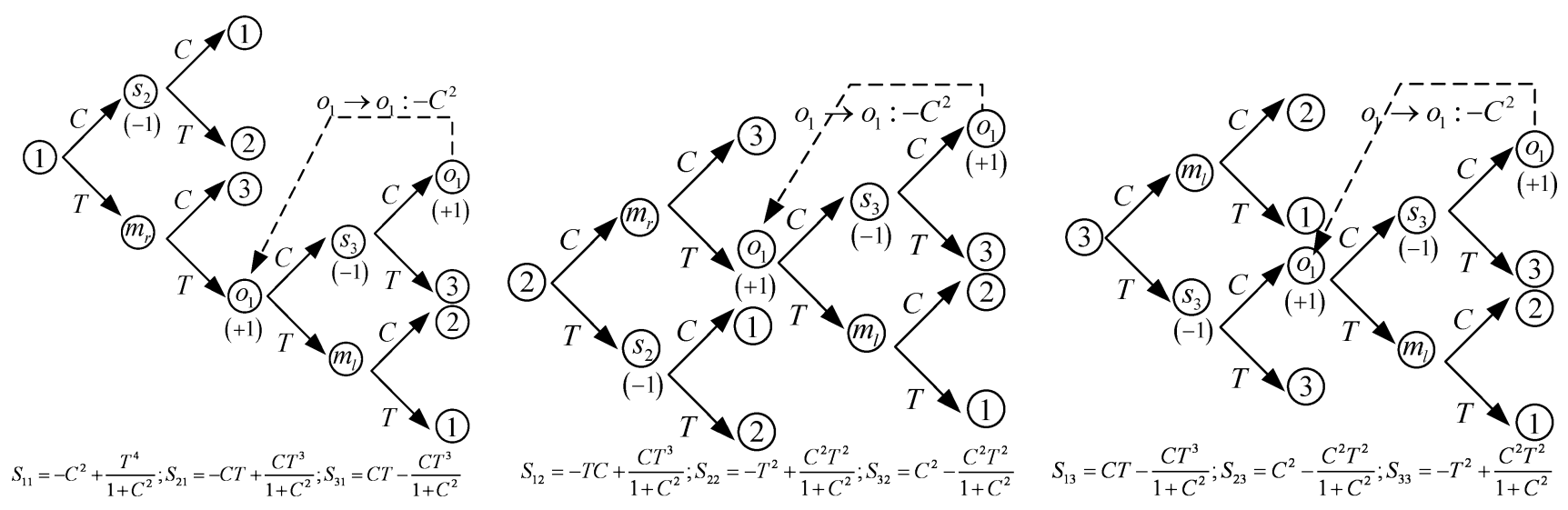

Fig. 3. $S$-parameter derivation of the planar Marchand balun. The nodes (1-3) of the tree denote ports 1-3. The others nodes are denoted in Fig. 1.

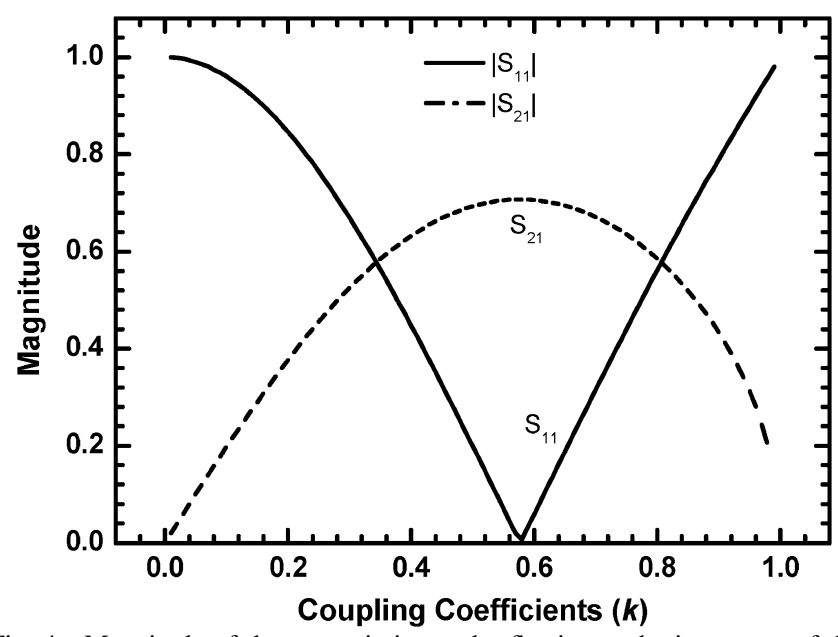

Fig. 4. Magnitude of the transmission and reflection at the input port of the planar Marchand balun with respect to coupling coefficients $k$ for the cases of no loss.

to the symmetric signal delivery, as shown in Fig. 2(a) and (b). This procedure to figure out the scattering parameters can be portrayed in the tree formation, as shown in Fig. 3. The tree nodes $1,2,3, o_{1 \sim 3}$ and $s_{1 \sim 2}$, in Fig. 3, symbolize ports $1-3$, open end, and short terminal, respectively, as shown in Fig. 1. As a result, the $S$-parameter matrix of the planar coupled-line Marchand balun can be expressed as

$$
[S]=\left[\begin{array}{ccc}
-C^{2}+\frac{T^{4}}{1+C^{2}} & -C T+\frac{C T^{3}}{1+C^{2}} & C T-\frac{C T^{3}}{1+C^{2}} \\
-C T+\frac{C T^{3}}{1+C^{2}} & -T^{2}+\frac{C^{2} T^{2}}{1+C^{2}} & C^{2}-\frac{C^{2} T^{2}}{1+C^{2}} \\
C T-\frac{C T^{3}}{1+C^{2}} & C^{2}-\frac{C^{2} T^{2}}{1+C^{2}} & -T^{2}+\frac{C^{2} T^{2}}{1+C^{2}}
\end{array}\right] .
$$

Fig. 4 displays the magnitude of the transmission and reflection at the input port of two configurations with respect to different coupling coefficients $k$, as defined in the Appendix with the assumption of no loss. The coupling coefficient is designed as the value of $1 / \sqrt{3}$ or $-4.8 \mathrm{~dB}$, for a good input matching, i.e., $S_{11}=0$, and the maximum transmission [14]. The optimal Marchand balun is practically implemented on account of the low optimal coupling coefficient.

\section{B. Implementation}

The coupled-line Marchand balun can be realized by Lange couplers [15], [16], broadside coupled lines [12], [17], [18], and spiral transmission lines [12], [17], [19]-[21]. In order to shrink the size of the balun, an interleave transformer is employed as a quarter-wavelength coupled line in our study, as shown in Fig. 1. The transformer-type coupled lines, namely, spiral transmission lines, can achieve the desired coupling coefficient. The coupledline Marchand balun with two short terminals and one open end is applied and the two ac ground terminals tied together can provide a dc bias for the mixer's switch quad.

For the balanced mixers, the LO switch quad is driven by the differential signals. A wideband single-to-differential Marchand balun is demanded in order to offer differential LO signals and to reserve the mixer wideband operation. Given that the input impedance of the Gilbert cell $Z_{1}$ is not matched to the source impedance $Z_{0}$, the $S$-parameters of Marchand balun are modified as shown in (5) at the bottom of the following page [14]. However, the balance of the two outputs is independent of the coupling coefficient $k$ and the load impedance $Z_{1}$. Even if the load impedance of the Marchand balun is not matched, the outputs also have equal magnitude and opposite phase.

Most monolithic Marchand baluns are fabricated on the semi-insulating GaAs substrate. A Marchand balun on the high-resistivity ( $>4000 \Omega \cdot \mathrm{cm}$ ) silicon substrate had also been demonstrated [22]. Recently, the Marchand balun was practiced using standard silicon processing with a shielding ground plane [23]. However, the shielding ground plane limits the even-mode characteristic impedance and then reduces the balun bandwidth [21]. The operating bandwidth of the Marchand balun increases monotonically when the ratio of the even-mode characteristic impedance to the odd-mode characteristic impedance of the coupled line increases. A high even-mode characteristic impedance is preferred for a wideband Marchand balun. Thus, the high even-mode characteristic impedance of coupled lines can be achieved in our Marchand balun topology to obtain wide bandwidth. Besides, the higher effective dielectric constant for the balun without the shielding ground plane is good for size reduction.

In this paper, the planar Marchand balun, as shown in Fig. 5, is implemented directly on the low-resistivity $(\sim 10 \Omega \cdot \mathrm{cm})$ silicon 


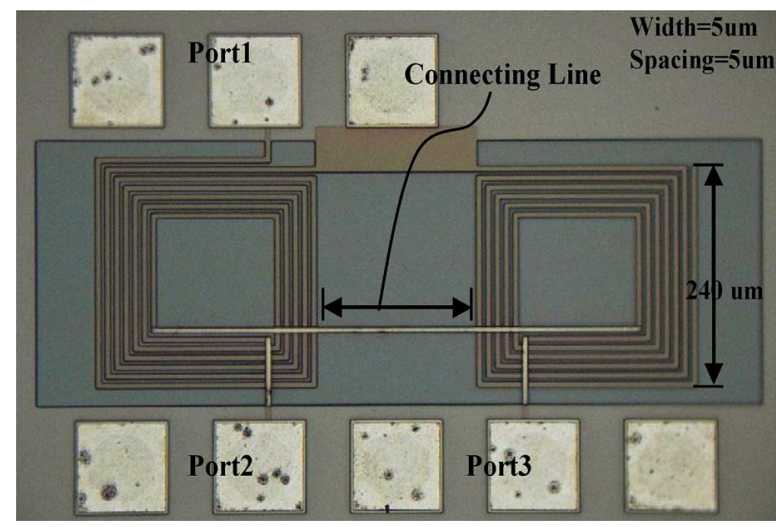

Fig. 5. Die photograph of a monolithic Marchand balun. The connecting line is approximately $180 \mu \mathrm{m}$ and is restricted by the GSGSG probe. From simulation, the coupling coefficient of the coupled line is approximately 0.5 at $12 \mathrm{GHz}$. (Color version available online at http://ieeexplore.ieee.org.)

substrate with the high even-mode characteristic impedance to hold broadband operation. This Marchand balun is formed by two-section transformer-type coupled lines and is designed at the center frequency of $12 \mathrm{GHz}$. The size of Marchand balun is approximately $660 \mu \mathrm{m} \times 250 \mu \mathrm{m}$. It is very compact thanks to the size advantage of the transformer-type coupled lines. The coupled lines are made of the top metal with the thickness of $0.93 \mu \mathrm{m}$, the spacing of $5 \mu \mathrm{m}$, and the width of $5 \mu \mathrm{m}$. The interleave transformer has approximately $3: 3$ turns. The substrate thickness is approximately $350 \mu \mathrm{m}$ and the distance between the top metal and the substrate is approximately $6.2 \mu \mathrm{m}$. From simulation, the coupling coefficient of the coupled line is approximately 0.5 at $12 \mathrm{GHz}$. The experimental measured data, IE3D simulation results, and the calculated data from (4) based on IE3D simulated $C$ and $T$ of the Marchand balun in Fig. 5 are displayed in Figs. 6 and 7. The delta plots of the phase and amplitude errors are presented in Fig. 8. The magnitude imbalance in output ports is approximately $2 \mathrm{~dB}$. This magnitude imbalance results from the loss of the connecting line between two transformer-type coupled lines, as shown in Fig. 5. The length of the connecting line is approximately $180 \mu \mathrm{m}$. The inevitable finite connecting line in the Marchand balun test pattern is constrained by the ground-signal-ground-signal-ground

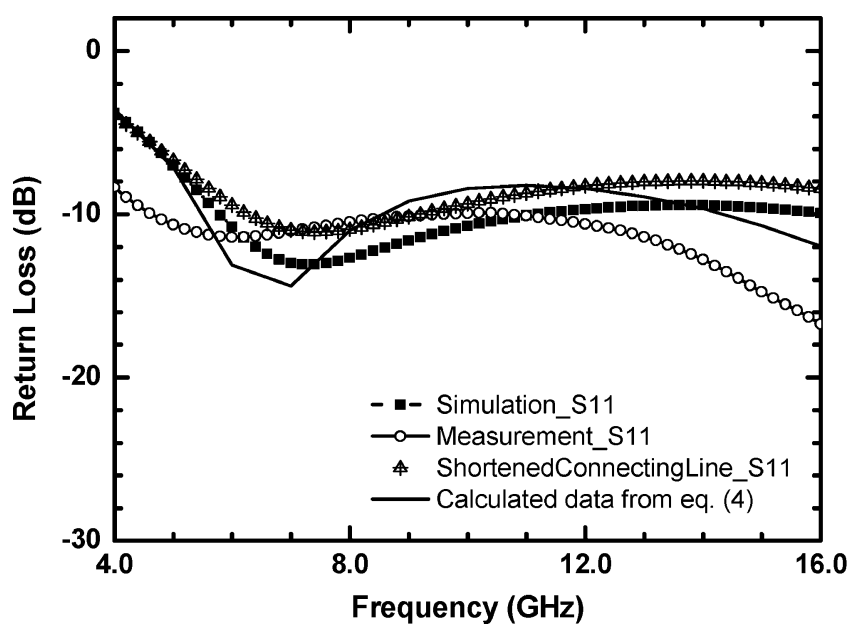

Fig. 6. Input return loss of the planar Marchand balun.

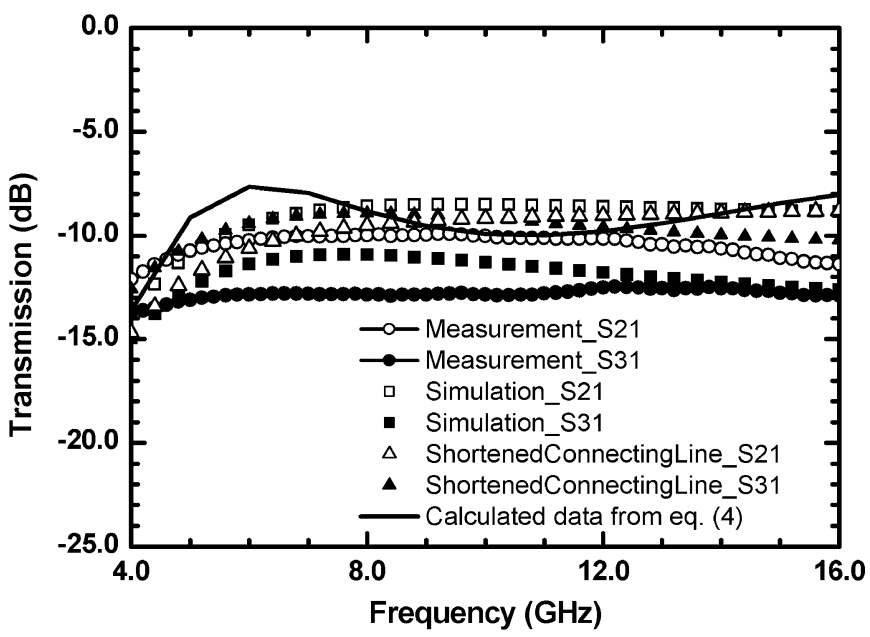

Fig. 7. Transmission coefficients of the planar Marchand balun.

(GSGSG) pad employed for the measurement purpose. The finite connecting line can be minimized in the final fabricated circuit. On the low-resistivity silicon substrate, the signal transmission of the balun is dominated by the first component, i.e., the direct coupled term $C \times T$ of (2) and (3). However, the voltage

$$
[S]=\left[\begin{array}{ccc}
\frac{1-k^{2}\left(\frac{2 Z_{1}}{Z_{0}}+1\right)}{1+k^{2}\left(\frac{2 Z_{1}}{Z_{0}}-1\right)} & j \frac{2 k \sqrt{1-k^{2}} \sqrt{\frac{Z_{1}}{Z_{0}}}}{1+k^{2}\left(\frac{2 Z_{1}}{Z_{0}}-1\right)} & -j \frac{2 k \sqrt{1-k^{2}} \sqrt{\frac{Z_{1}}{Z_{0}}}}{1+k^{2}\left(\frac{2 Z_{1}}{Z_{0}}-1\right)} \\
j \frac{2 k \sqrt{1-k^{2}} \sqrt{\frac{Z_{1}}{Z_{0}}}}{1+k^{2}\left(\frac{2 Z_{1}}{Z_{0}}-1\right)} & \frac{1-k^{2}}{1+k^{2}\left(\frac{2 Z_{1}}{Z_{0}}-1\right)} & j \frac{2 k^{2} \sqrt{\frac{Z_{1}}{Z_{0}}}}{1+k^{2}\left(\frac{2 Z_{1}}{Z_{0}}-1\right)} \\
-j \frac{2 k \sqrt{1-k^{2}} \sqrt{\frac{Z_{1}}{Z_{0}}}}{1+k^{2}\left(\frac{2 Z_{1}}{Z_{0}}-1\right)} & j \frac{2 k^{2} \frac{Z_{1}}{Z_{0}}}{1+k^{2}\left(\frac{2 Z_{1}}{Z_{0}}-1\right)} & \frac{1-k^{2}\left(\frac{2 Z_{1}}{Z_{0}}-1\right)}{\left.1+k^{2}\right)}
\end{array}\right]
$$




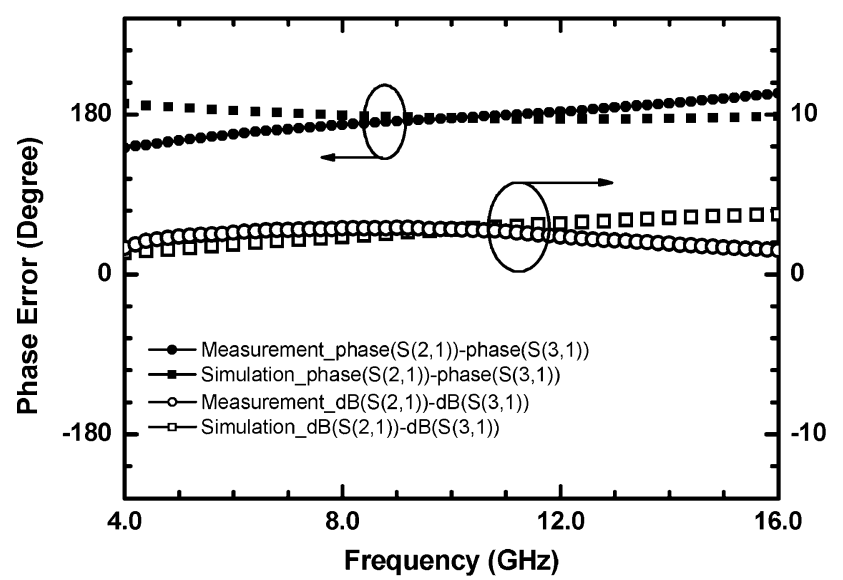

Fig. 8. Phase error and magnitude error of the planar Marchand balun.

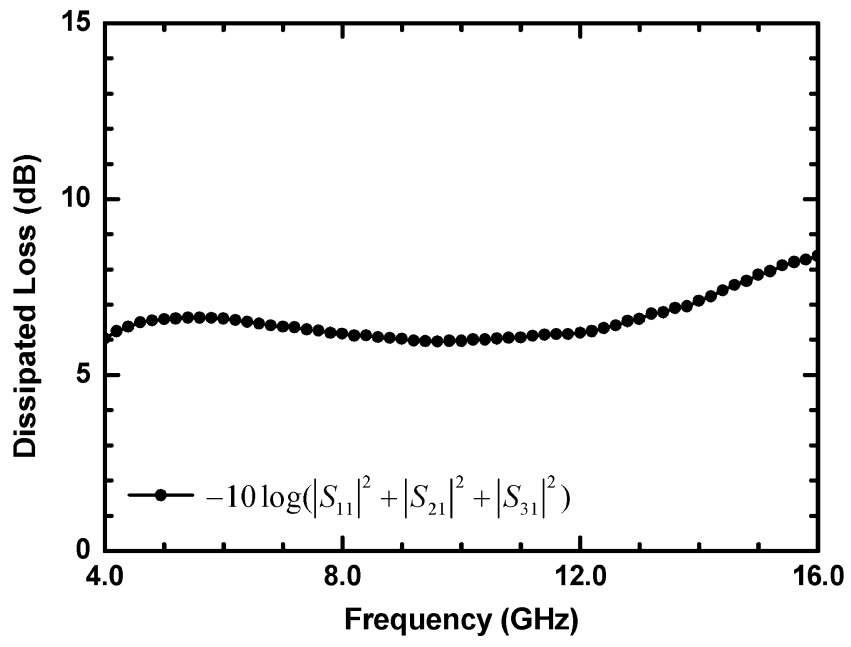

Fig. 9. Dissipated loss of the Marchand balun.

wave directly coupled to port 3 experiences the connecting-line loss. Thus, the transmission magnitude $\left|S_{31}\right|$ is lower than $\left|S_{21}\right|$. This phenomenon corresponds to the measured results. The outputs are more balanced in magnitude when the connecting line is removed in the IE3D simulation, as shown in Fig. 7, but the phase balance is almost unaffected by the connecting line. In other words, the connecting line has high associated loss than phase delay. The dissipated loss of a Marchand balun is defined as

$$
\text { Loss }=-10 \log \left(\left|S_{11}\right|^{2}+\left|S_{21}\right|^{2}+\left|S_{31}\right|^{2}\right)
$$

and is approximately $6 \mathrm{~dB}$, as shown in Fig. 9. In our study, the Gilbert mixer with the integrated Marchand balun has a short connecting line to provide balanced outputs. The usable bandwidth is more than $10 \mathrm{GHz}$.

The mixer conversion gain is insensitive to the LO power provided that the phase is balanced and the LO power is large enough to commutate the RF current. The reason will explained by the measured results in Section V. The magnitude imbalance resulting from the small connecting line loss is, hence, not a matter of mixer's operation. This balun is appropriately utilized as a single-to-differential balun at the LO port in this mixer even though the magnitude imbalance occurs.

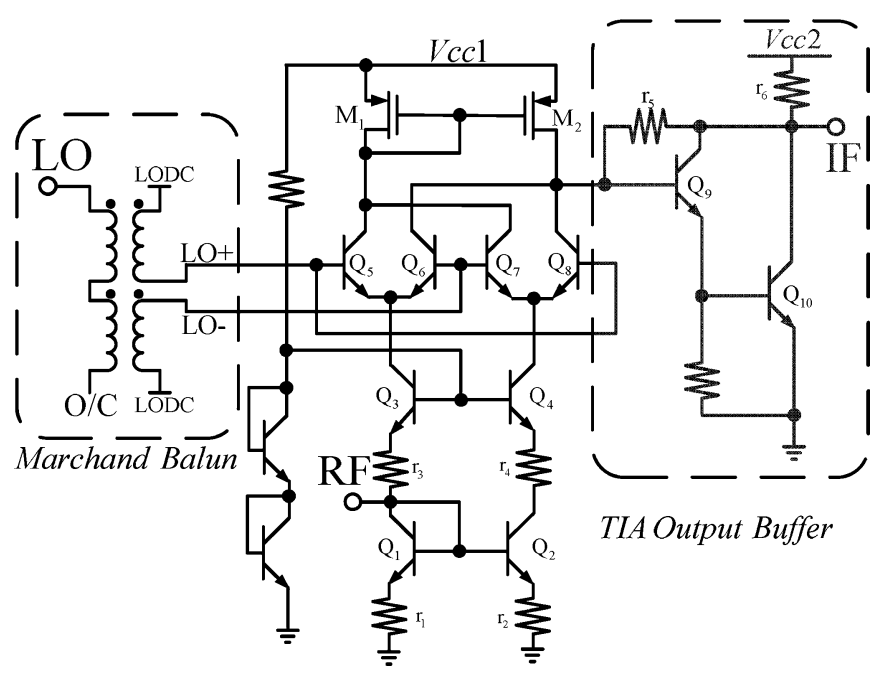

Fig. 10. Schematic of the micromixer with an LO Marchand balun and a TIA output buffer.

\section{CirCUit DESIGN}

The entire schematic of the single-ended wideband downconverter is shown in Fig. 10. This downconverter is formed by the micromixer, the Marchand balun, and the TIA output buffer. Each element has the broadband property.

The micromixer can be considered as the combination of two single-balanced mixers. One mixer is formed by the commonemitter-configured RF amplifier $Q_{2}$; the other is composed of the common-base-configured RF amplifier $Q_{3}$. The LO switch quad is made up of the transistors $Q_{5}, Q_{6}, Q_{7}$, and $Q_{8}$. The current mirror pair $Q_{1}$ and $Q_{2}$ provides the balance dc currents in the RF input stage and then these two RF amplifiers have equal magnitude and opposite phase transconductance gain to obtain good mixer balance. Moreover, the diode-type transistor $Q_{1}$ reduces the input impedance of $Q_{2}$ and enhances the speed of the common-emitter-configured input stage. The input impedance is controlled by the transistors $Q_{1}$ and $Q_{3}$ and the resistors $r_{1}$ and $r_{3}$. It is easy to achieve wideband matching so this micromixer can act as a wideband mixer [24].

To establish a single-ended output, the pMOS current mirror is applied to combine the differential output current signals of the mixer. Furthermore, a TIA amplifier is used in the output stage of this mixer. The frequency response of the input stage is dominated by the common-emitter-configured transistor $Q_{2}$. As shown in Fig. 11, in the critical path, the RF input stage is viewed as a transconductance amplifier (TCA), the IF output stage is a TIA, while the LO switch quad is inserted in the middle and performs the frequency translation. The topology is very similar to the well-known Cherry-Hooper amplifier-a TCA stage in cascade with a TIA stage [7]. The LO current commutation quad Gilbert mixer cell is used to switch the connecting current between the TCA and TIA stages. Thus, the conversion gain and frequency response can be analyzed as a TCA for the RF stage and a TIA for the IF stage. The TIA output buffer employs a resistive feedback to enlarge the output bandwidth. In addition, a Darlington pair is also utilized to enhance the speed of transistors. Therefore, this output stage of the mixer 


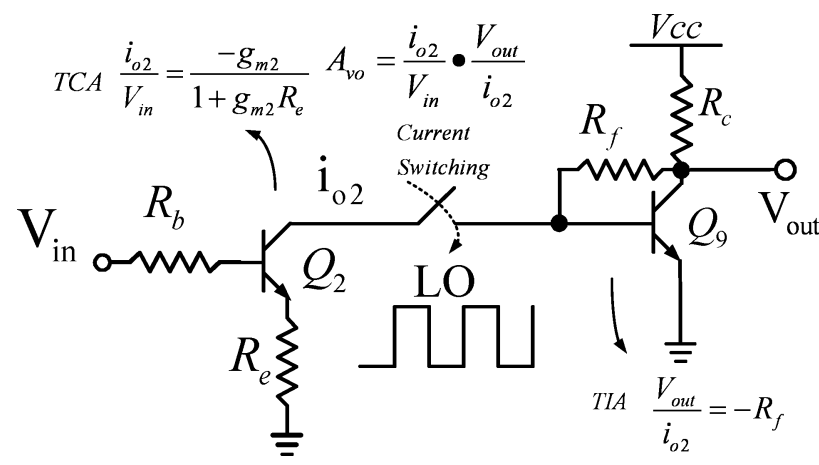

Fig. 11. Cherry-Hooper technique employed in the micromixer.

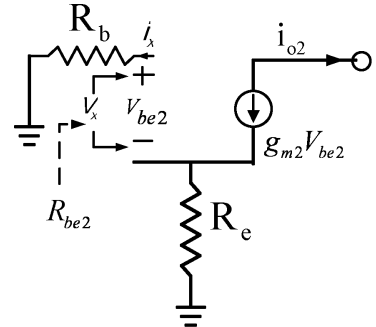

(a)

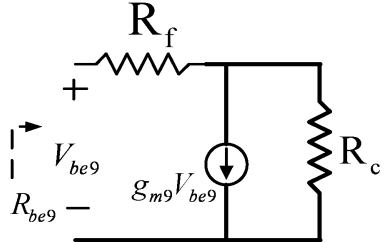

(b)
Fig. 12. Small-signal models of the: (a) TCA and (b) TIA

has single-ended and wideband properties. To analyze the frequency response, the small-signal model is split into two parts, as shown in Fig. 12. For simplicity, the base-emitter resistance and base-collector capacitance are neglected in the frequency response analysis. The complete transfer functions of the TCA and TIA stages from exact circuit analysis are denoted as

$$
A_{v}(S)=\frac{i_{o 2}}{V_{\text {in }}}(S) \cdot \frac{V_{\text {out }}}{i_{o 2}}(S)=\frac{G_{m 2}}{1+S / \omega_{P 2}} \times \frac{R_{m 9}}{1+S / \omega_{P 9}}
$$

where

$$
\left\{\begin{array}{l}
G_{m 2}=\frac{g_{m 2}}{1+g_{m 2} R_{e}} \\
R_{m 9}=-R_{f} \\
\omega_{P 2}=\frac{1}{C_{\pi 2} \frac{R_{e}+R_{b}}{1+g_{m 2} R_{e}}} \\
\omega_{P 9}=\frac{1}{C_{\pi 9} \frac{R_{f}+R_{c}}{1+g_{m 9} R_{c}}} .
\end{array}\right.
$$

From the open-circuit time-constant analysis, the poles of the TCA and TIA stages in Fig. 12 are

and

$$
\omega_{P 2}=\frac{1}{R_{b e 2} C_{\pi 2}}
$$

$$
\omega_{P 9}=\frac{1}{R_{b e 9} C_{\pi 9}}
$$

where

$$
R_{b e 2}=\frac{R_{e}+R_{b}}{1+g_{m 2} R_{e}}
$$

and

$$
R_{b e 9}=\frac{R_{f}+R_{c}}{1+g_{m 9} R_{c}}
$$

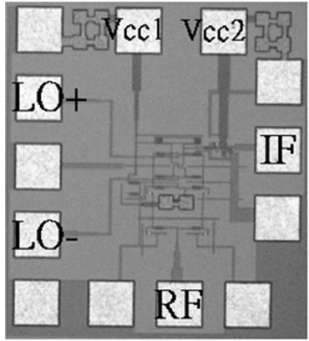

(a)

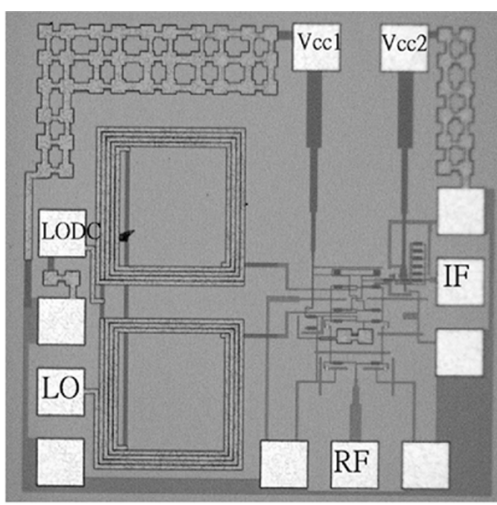

(b)
Fig. 13. Die photographs of the micromixers: (a) without a Marchand balun and (b) with a Marchand balun designed at the center frequency of $9 \mathrm{GHz}$.

are the resistances looking into the base-emitter terminals of $Q_{2}$ and $Q_{9}$ in Fig. 12, respectively [25]. As a result of a resistive feedback, the poles are extended by the feedback factor of $1+$ $g_{m} R$. The overall voltage gain can be calculated as

$$
A_{\mathrm{vo}}=G_{m 2} \cdot R_{m 9} \simeq \frac{g_{m 2} R_{f}}{1+g_{m 2} R_{e}} .
$$

These three components (the micromixer, TIA amplifier, and Marchand balun) construct a single-ended wideband downconverter. Due to the single-ended and wideband matching properties, this Marchand micromixer with the integrated Marchand balun has a wide range of usage.

\section{Measurement Results}

The Gilbert mixer along with a compensated Marchand balun was demonstrated on a semi-insulating GaAs substrate by Hamed et al. [26]. In this paper, the coupled-line planar Marchand balun is implemented on a low-resistivity standard silicon substrate and, to the best of our knowledge, combined with a micromixer for the first time. Two wideband micromixers were fabricated using 0.35- $\mu \mathrm{m} \mathrm{SiGe} \mathrm{BiCMOS} \mathrm{technology} \mathrm{[27].}$ Both mixers have identical active circuitry, except the LO Marchand balun. Fig. 13 illustrates two die photographs of the implemented mixers. The left-hand-side photograph is a micromixer without a Marchand balun and its chip size is to approximately $0.75 \mathrm{~mm} \times 0.75 \mathrm{~mm}$. The chip size of the right-hand-side chip with an integrated Marchand balun is approximately $1 \mathrm{~mm} \times 1 \mathrm{~mm}$. This integrated Marchand balun is redesigned by taking away the connecting line described in Fig. 5 in order to obtain more balanced outputs. The Marchand balun only occupies the area of $300 \mu \mathrm{m} \times 700 \mu \mathrm{m}$ and is designed at the center frequency of $9 \mathrm{GHz}$. The measurement results of the micromixer without the Marchand balun give excellent agreement with the wideband operation of the micromixer. The Marchand mixer then also performs wideband mixing.

\section{A. Micromixer Without a Marchand Balun}

The mixer without the Marchand balun has the broad band property and works up to $15 \mathrm{GHz}$. The conversion gain is ap- 


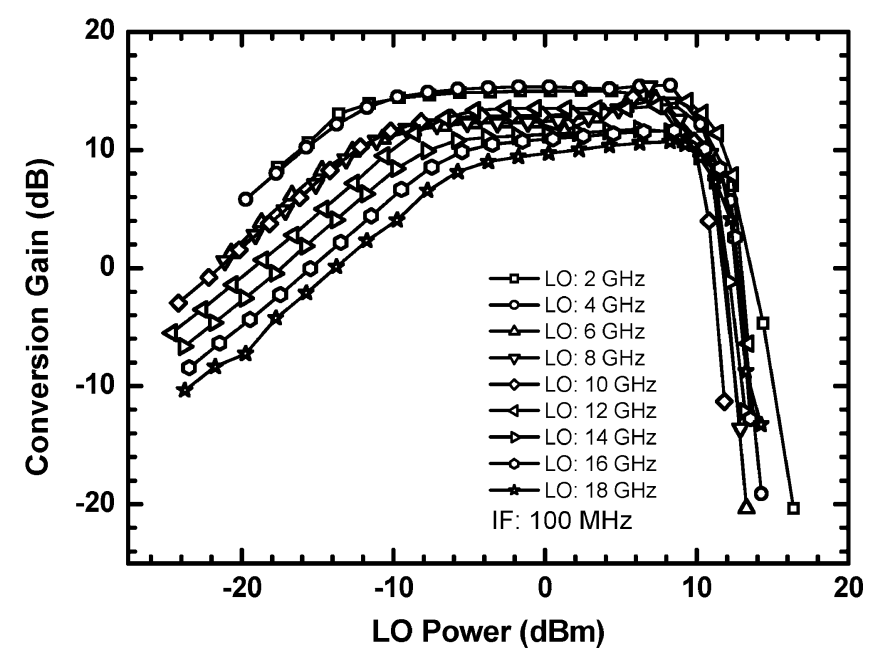

Fig. 14. Conversion gain of the micromixer without Marchand balun with respect to LO power.

proximately $15 \mathrm{~dB}$ and the 3 -dB IF bandwidth is approximately $400 \mathrm{MHz}$ [27].

Fig. 14 illustrates that the effect of the LO power on the conversion gain is measured with the fixed IF frequency of $100 \mathrm{MHz}$, but different LO frequencies by Agilent's E4448A power spectrum analyzer (PSA). The current commutation in the Gilbert cell is responsible for the frequency translation. The differential pair of the bipolar Gilbert cell only needs a small twist voltage (approximately $0.1 \mathrm{~V}$ ) to perform the near-perfect current commutation. Once the LO power is large enough to drive the switch quad of the Gilbert mixer, the conversion gain keeps constant and is insensitive to the LO power. If the LO power is too large, the quad switch SiGe HBT transistors enter the saturation region and then the mixer gain degrades. However, there is more power to drive the switch quad at higher frequencies. With the LO frequency increase, the LO power range for the flat constant conversion gain region decreases.

The IF and RF bandwidth experiments in [27] give the direct measurement of the IF and RF stage frequency response of the mixer, while the frequency response of the LO Gilbert cell is examined in Fig. 14. The higher the LO frequency, the narrow the flat gain region becomes. The flat gain region still exists for LO frequencies up to $18 \mathrm{GHz}$. According to the measured results, the maximum operating frequency of the mixer is approximately $15 \mathrm{GHz}$ and is limited by the RF input stage.

\section{B. Micromixer with an Integrated Marchand Balun}

To form a single-ended mixer, a Marchand balun is utilized at the LO port. This Marchand balun has more than $10-\mathrm{GHz}$ bandwidth and is compatible with the wideband property of the micromixer. The measured return loss at the RF, LO, and IF ports is represented in Fig. 15. The return-loss performance of the RF input and IF output keeps the same as that of the previous active mixer, while the return loss of the LO input is improved by the Marchand balun. The return loss of the RF, LO, and IF ports is below $-14,-6$, and $-10 \mathrm{~dB}$, respectively. The conversion gain of the micromixer with the LO Marchand balun is

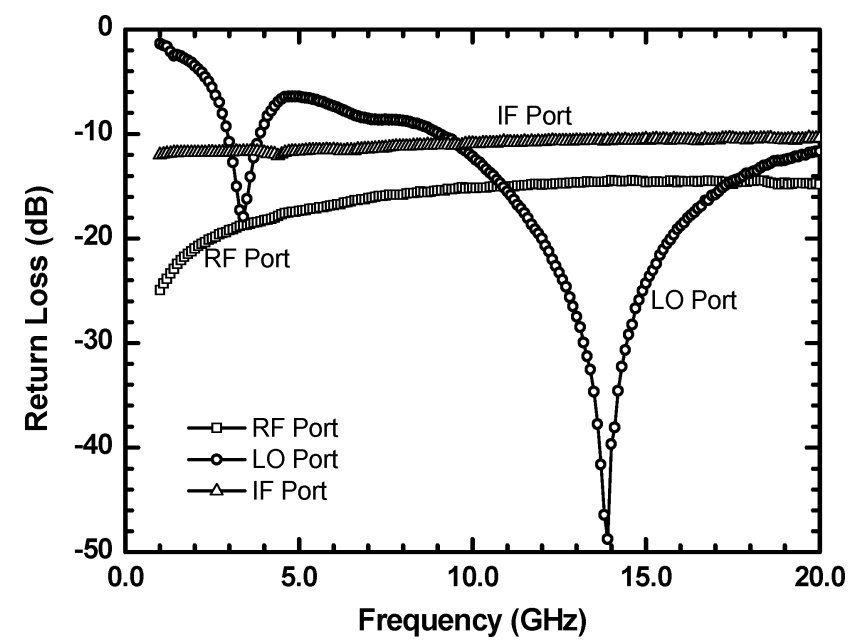

Fig. 15. Return loss of the micromixer with the integrated planar Marchand balun.

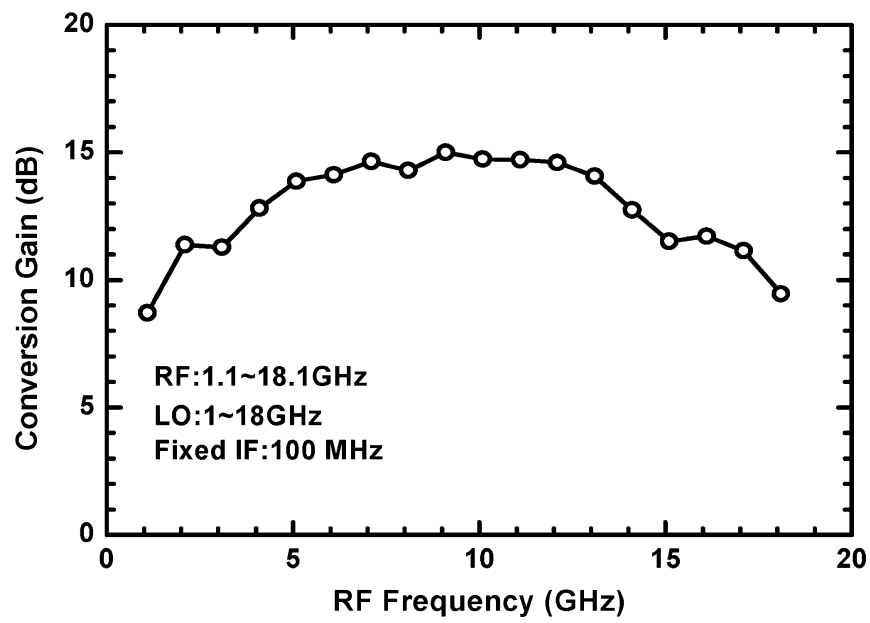

Fig. 16. Conversion gain of the micromixer with the integrated planar Marchand balun.

measured with a fixed 100-MHz IF when the LO power equals to $6 \mathrm{~dB}$. Fig. 16 displays the experiment result. This mixer with the integrated Marchand balun can operate from 3.5 to $14.5 \mathrm{GHz}$ with 11-GHz 3-dB bandwidth. The conversion gain in the 3-dB bandwidth is approximately $15 \mathrm{~dB}$ and is the same as that of the mixer without the Marchand balun.

The low-resistivity substrate of standard silicon IC process introduces the connecting line loss. Even if this loss causes the output magnitude imbalance of the Marchand balun, the magnitude imbalance can be tolerable when a Marchand balun is employed at the LO part of mixer as a single-to-different balun because LO power does not affect the conversion gain at the flat region, as shown in Fig. 14. The switch quad SiGe HBT transistors especially only demand small power to steer the RF current totally from one side to the other side of the differential pair. In other words, there is a wide range of the constant conversion gain region in terms of the LO power. Thus, the wideband Marchand balun is properly used to preserve the wideband mixing even if there is magnitude imbalance. The $3.5-\mathrm{GHz}$ lower bound and $14.5-\mathrm{GHz}$ upper bound of the mixer's frequency response 


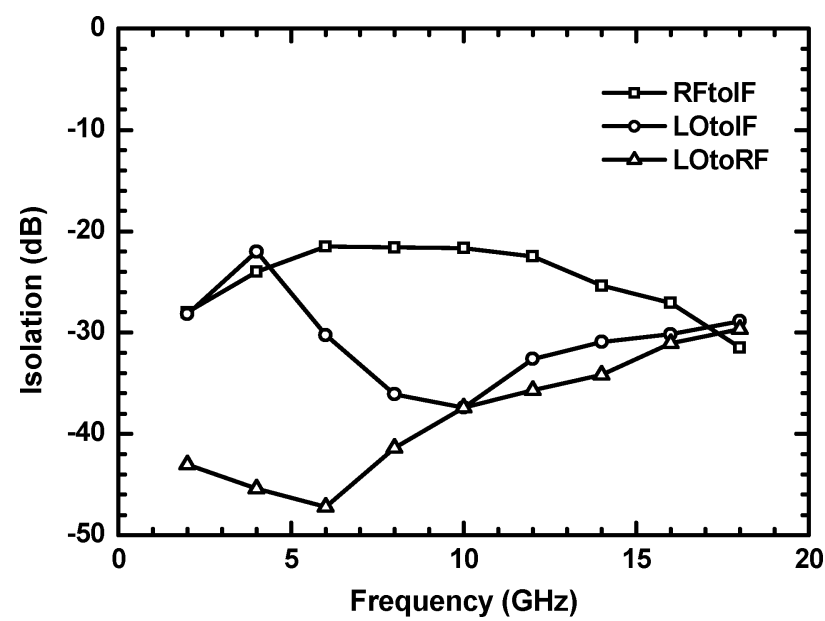

Fig. 17. Port-to-port isolations of the micromixer with the integrated planar Marchand balun.

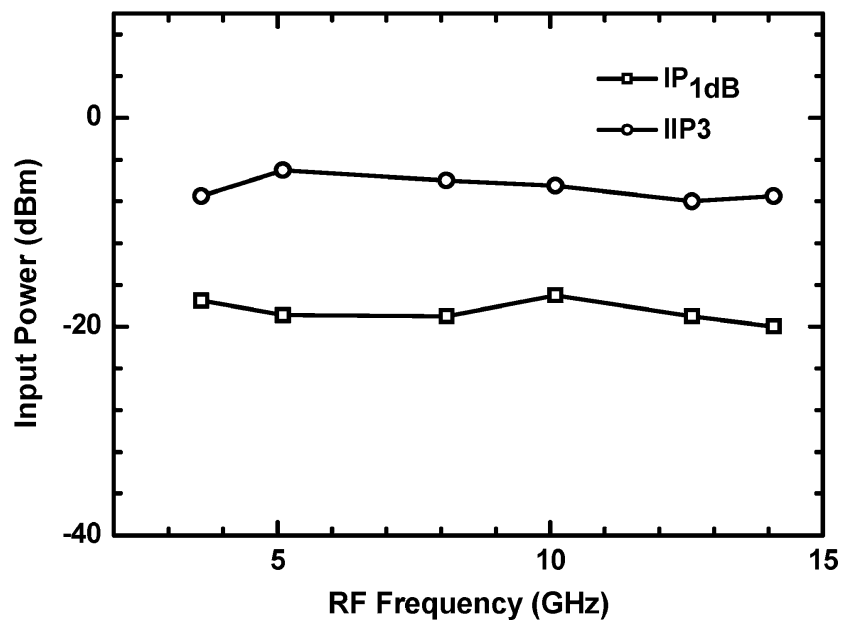

Fig. 18. $\operatorname{IP}_{1 \mathrm{~dB}}$ and $\operatorname{IIP}_{3}$ of the micromixer with the integrated planar Marchand balun.

are restricted by the Marchand balun and RF input stage, respectively, according to the measured results of the individual components (the Marchand balun and Gilbert mixer without balun) and the experimental outcome in Fig. 16.

The port-to-port isolations of the Marchand mixer are presented in Fig. 17. During the operating frequencies of the Marchand balun, the mixer has the higher LO-to-IF isolation of approximately $35 \mathrm{~dB}$. In addition, the reverse isolation of the transistors at the input stage provides the higher LO-to-RF isolation, especially at low frequencies. The RF-to-IF isolation is below $-20 \mathrm{~dB}$.

The input 1-dB gain compression point $\mathrm{IP}_{1 \mathrm{~dB}}$ and the input third-order intercept point $\mathrm{IIP}_{3}$ of the micromixer with the integrated Marchand balun as a function of frequency are measured, as shown in Fig. 18. $\mathrm{IP}_{1 \mathrm{~dB}}$ is approximately $-19 \mathrm{dBm}$, while $\mathrm{IIP}_{3}$ is approximately $-7 \mathrm{dBm}$. The input signal with the frequency below $7 \mathrm{GHz}$ has harmonics located in the operating frequency range. Hence, the in-band harmonic is measured when the input frequency is $5 \mathrm{GHz}$ and $\mathrm{IIP}_{2}$ is approximately $12 \mathrm{dBm}$.

The noise figure of the Marchand mixer is measured at each frequency of 2, 6, 10, and $14 \mathrm{GHz}$, as shown in Fig. 19. When the

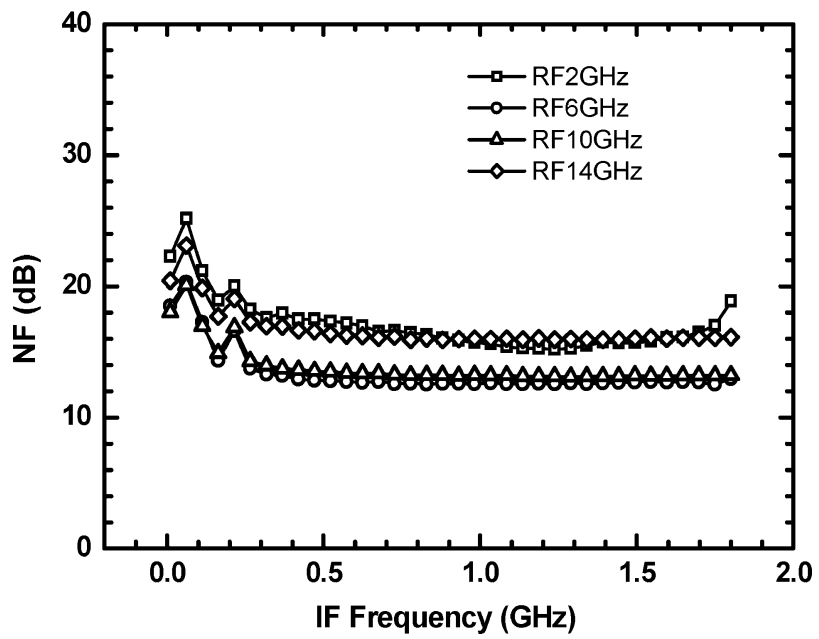

Fig. 19. Noise figure of the micromixer with the integrated planar Marchand balun.

mixer operates at 6 and $10 \mathrm{GHz}$, the conversion gain is higher and the noise figure is lower simultaneously. The lowest noise figure is approximately $13 \mathrm{~dB}$.

Both mixers work with a 5-V supply and have the same core power consumption of approximately $60 \mathrm{~mW}$. The additional Marchand balun does not consume any de power. This wideband Marchand micromixer not only maintains the performances of the original micromixer without an integrated balun, but also provides a single-ended input and output solution.

\section{CONCLUSION}

In this paper, a heuristic approach to derive the three-port scattering parameters of the lossy Marchand balun has been introduced. The appropriate Marchand balun topology with the capability of resisting the loss in the standard silicon IC process has been identified. A systematic approach to measure the frequency response of the RF, IF, and LO stages of a Gilbert mixer has been developed. Thus, a single-ended wideband Gilbert mixer with the integrated planar Marchand balun has been demonstrated using $0.35-\mu \mathrm{m}$ SiGe BiCMOS technology. This single-ended wideband mixer with the integrated Marchand balun has the conversion gain of $15 \mathrm{~dB}, \mathrm{IP}_{1 \mathrm{~dB}}$ of $-19 \mathrm{dBm}$, $\mathrm{IIP}_{3}$ of $-7 \mathrm{dBm}, \mathrm{IIP}_{2}$ of $12 \mathrm{dBm}$, a minimum noise figure of $13 \mathrm{~dB}$, and works from 3.5 to $14.5 \mathrm{GHz}$ with $400-\mathrm{MHz}$ IF bandwidth. The developed frequency response measurement technique can be employed to identify the frequency-limiting mechanism. The lower bound of $3.5 \mathrm{GHz}$ is limited by the LO stage, while the upper bound of $14.5 \mathrm{GHz}$ is limited by the RF stage.

\section{APPENDIX}

\section{DERIVATION OF COUPLED AND TRANSMISSION SCATTERINC PARAMETERS OF A LOSSY COUPLED LINE}

The even- and odd-mode $A B C D$ matrix method is employed to analyze the lossy coupled line, as shown in Fig. 20. Half of the coupled line can be considered as a lossy transmission line. Then, the even- and odd-mode $A B C D$ matrices are denoted as 


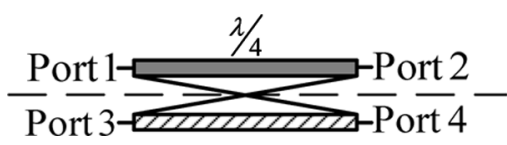

Fig. 20. Four-port network of coupled lines.

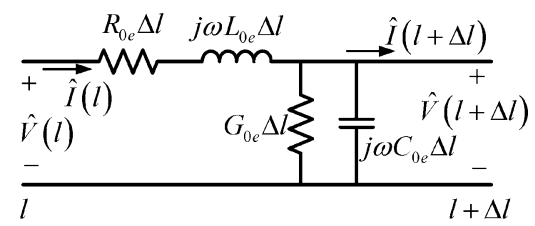

Fig. 21. Electric circuit model of a transmission line for the even mode.

$$
\left[\begin{array}{ll}
A & B \\
C & D
\end{array}\right]_{e}=\left[\begin{array}{cc}
\cosh (\gamma \ell) & \hat{Z}_{0 e} \sinh (\gamma \ell) \\
\hat{Y}_{0 e} \sinh (\gamma \ell) & \cosh (\gamma \ell)
\end{array}\right]
$$

and

$$
\left[\begin{array}{ll}
A & B \\
C & D
\end{array}\right]_{o}=\left[\begin{array}{cc}
\cosh (\gamma \ell) & \hat{Z}_{0 o} \sinh (\gamma \ell) \\
\hat{Y}_{0 o} \sinh (\gamma \ell) & \cosh (\gamma \ell)
\end{array}\right]
$$

respectively. A lossy transmission line for the even mode is shown in Fig. 21 and the even-mode characteristic impedance is expressed as

$$
\hat{Z}_{0 e}=\sqrt{\left(R_{0 e}+j \omega L_{0 e}\right) /\left(G_{0 e}+j \omega C_{0 e}\right)}
$$

with the per-unit-length resistance $R_{0 e}$, inductance $L_{0 e}$, conductance $G_{0 e}$, and capacitance $C_{0 e}$, [28]. $\hat{Z}_{0 o}$ is the odd-mode characteristic impedance and can be defined similarly to $\hat{Z}_{0 e}$ with its associated $R, L, G$, and $C$. The even- and odd-mode propagation constants are assumed to be equal for simplicity and are defined as $\gamma$. In other words, $\hat{Z}_{0 e}$ and $\hat{Z}_{0 o}$ are complex numbers for the lossy transmission line. Under the matching condition of $\hat{Z}_{0 e} \hat{Z}_{0 o}=Z_{0}^{2}$, where $Z_{0}$ is the terminal impedance, the coupled line has perfect matching and excellent isolation. Therefore, the scattering parameter matrix for a lossy coupled line is derived as

$$
[S]=\left[\begin{array}{llll}
0 & T & C & 0 \\
T & 0 & 0 & C \\
C & 0 & 0 & T \\
0 & C & T & 0
\end{array}\right]
$$

where the scattering parameters for the coupled and transmitted ports $C$ and $T$ are

$$
T=\frac{2}{2 \cosh (\gamma \ell)+\left(\frac{\hat{Z}_{0 e}}{Z_{0}}+\frac{Z_{0}}{\hat{Z}_{0 e}}\right) \sinh (\gamma \ell)}
$$

and

$$
C=\frac{\left(\frac{\hat{Z}_{0 e}}{Z_{0}}-\frac{Z_{0}}{\hat{Z}_{0 e}}\right) \sinh (\gamma \ell)}{2 \cosh (\gamma \ell)+\left(\frac{\hat{Z}_{0 e}}{Z_{0}}+\frac{Z_{0}}{\hat{Z}_{0 e}}\right) \sinh (\gamma \ell)}
$$

respectively [29]. The Marchand balun analysis in Section II-A is developed based on this scattering matrix (A4). As shown in (A3), the lossy characteristic impedances $\hat{Z}_{0 e}$ and $\hat{Z}_{0 o}$ vary with frequencies in the presence of loss and the coupled line is dispersive. In our analysis of the Marchand balun formula on a standard lossy silicon substrate in Section II, the values of $C$ and $T$ can be substituted by (A5) and (A6). The matching condition of $\hat{Z}_{0 e} \hat{Z}_{0 o}=Z_{0}^{2}$ is designed at the center frequency. Thus, the heuristic analysis, as shown in Figs. 2 and 3, is valid at the center frequency for a Marchand balun on a lossy substrate. Under the lossless condition, the complex characteristic impedances $\hat{Z}_{0 e}$ and $\hat{Z}_{0 o}$ become real numbers $Z_{0 e}$ and $Z_{0 o}$, respectively. The matching condition $Z_{0 e} Z_{0 o}=Z_{0}^{2}$ can be designed for all frequencies and, thus, the analysis in Figs. 2 and 3 is valid for all frequencies. At the frequency where $\beta l=\pi / 2$, (A5) and (A6) become well-known formulas as

$$
T=-j \sqrt{1-k^{2}}
$$

and

$$
C=k
$$

where the coupling coefficient $k$ is equal to

$$
k=\frac{Z_{0 e}-Z_{0 o}}{Z_{0 e}+Z_{0 o}} .
$$

\section{REFERENCES}

[1] K. R. Rao, J. Wilson, and M. Ismail, "A CMOS RF front-end for a multistandard WLAN receiver," IEEE Microw. Wireless Compon. Lett., vol. 15, no. 5, pp. 321-323, May 2005.

[2] P. Heydari, "A study of low-power ultra wideband radio transceiver architectures," in IEEE Wireless Commun. Networking Conf., Mar. 2005, vol. 2, pp. 758-763.

[3] A. Ghosh, D. R. Wolter, J. G. Andrews, and R. Chen, "Broadband wireless access with WiMax/802.16: Current performance benchmarks and future potential," IEEE Commun. Mag., vol. 43, no. 2, pp. 129-136, Feb. 2005.

[4] B. Gilbert, "The MICROMIXER: A highly linear variant of the Gilbert mixer using a bisymmetric class-AB input stage," IEEE J. Solid-State Circuits, vol. 32, no. 9, pp. 1412-1423, Sep. 1997.

[5] W. R. Brinlee, A. M. Pavio, and K. R. Varian, "A novel planar doublebalanced 6-18 GHz MMIC mixer," in IEEE MTT-S Int. Microw. Symp. Dig., San Diego, CA, May 1994, pp. 9-12.

[6] S. A. Maas and Y. Ryu, "A broadband, planar, monolithic resistive frequency doubler," in IEEE MTT-S Int. Microw. Symp. Dig., San Diego, CA, May 1994, vol. 1, pp. 443-446.

[7] E. M. Cherry and D. E. Hooper, "The design of wideband transistor feedback amplifiers," Proc. IEE, vol. 110, no. 2, pp. 375-389, Feb. 1963.

[8] B. Razavi, Design of Integrated Circuits for Optical Communications. New York: McGraw-Hill, 2003, ch. 5, sec. 5.2.3, pp. 136-140.

[9] N. Marchand, "Transmission-line conversion transformers," Electronics, vol. 17, no. 12, pp. 142-145, 1944.

[10] G. Oltman, "The compensated balun," IEEE Trans. Microw. Theory Tech., vol. 14, no. 3, pp. 112-119, Mar. 1966.

[11] A. M. Pavio and A. Kikel, "A monolithic or hybrid broadband compensated balun," in IEEE MTT-S Int. Microw. Symp. Dig., May 1990, vol. 1, pp. 483-486.

[12] T. Gokdemir, S. B. Economides, A. Khalid, A. A. Rezazadeh, and I. D. Robertson, "Design and performance of GaAs MMIC CPW baluns using overlaid and spiral couplers," in IEEE MTT-S Int. Microw. Symp. Dig., Denver, CO, Jun. 1997, pp. 401-404.

[13] R. Mongia, I. Bahl, and P. Bhartia, RF and Microwave Coupled-Line Circuits. Norwood, MA: Artech House, 1999, pp. 411-438.

[14] K. S. Ang and I. D. Robertson, "Analysis and design of impedancetransforming planar Marchand baluns," IEEE Trans. Microw. Theory Tech., vol. 49, no. 2, pp. 402-406, Feb. 2001.

[15] C. Nguyen and D. Smith, "Novel miniaturised wideband baluns for MIC and MMIC applications," Electron. Lett., vol. 29, no. 12, pp. 1060-1061, Jun. 1993. 
[16] M. C. Tsai, "A new compact wideband balun," in IEEE MTT-S Int. Microw. Symp. Dig., Jun. 1993, vol. 1, pp. 141-143.

[17] T.-H. Chen, K. W. Chang, S. B. Bui, H. Wang, G. S. Dow, L. C. T. Liu, T. S. Lin, and W. S. Titus, "Broadband monolithic passive baluns and monolithic double-balanced mixer," IEEE Trans. Microw. Theory Tech., vol. 39, no. 12, pp. 1980-1986, Dec. 1991.

[18] K. Nishikawa, I. Toyoda, and T. Tokumitsu, "Compact and broadband three-dimensional MMIC balun," IEEE Trans. Microw. Theory Tech., vol. 47, no. 1, pp. 96-98, Jan. 1999.

[19] Y. J. Yoon, Y. Lu, R. C. Frye, and P. R. Smith, "A silicon monolithic spiral transmission line balun with symmetrical design," IEEE Electron Device Lett., vol. 20, no. 4, pp. 182-184, Apr. 1999.

[20] K. S. Ang, S. B. Economides, S. Nam, and I. D. Robertson, "A compact MMIC balun using spiral transformers," in Asia-Pacific Microw. Conf., Singapore, Nov. 1999, pp. 655-658.

[21] M. Shimozawa, K. Itoh, Y. Sasaki, H. Kawano, Y. Isota, and O. Ishida, "A parallel connected Marchand balun using spiral shaped equal length coupled lines," in IEEE MTT-S Int. Microw. Symp. Dig., Anaheim, CA, Jun. 1999, pp. 1737-1740.

[22] Y. J. Yoon, Y. Lu, R. C. Frye, M. Y. Lau, P. R. Smith, L. Ahlquist, and D. P. Kossives, "Design and characterization of multilayer spiral transmission-line baluns," IEEE Trans. Microw. Theory Tech., vol. 47, no. 9, pp. 1841-1847, Sep. 1999.

[23] H.-Y. Chang, P.-S. Wu, T.-W. Huang, H. Wang, C.-L. Chang, and J. G. J. Chern, "Design and analysis of CMOS broadband compact highlinearity modulators for gigabit microwave/millimeter-wave applications," IEEE Trans. Microw. Theory Tech., vol. 54, no. 1, pp. 20-30, Jan. 2006.

[24] C. C. Meng, T. H. Wu, T. H. Wu, and G. W. Huang, "A 5.2 GHz 16 $\mathrm{dB}$ gain CMFB Gilbert downconversion mixer using $0.35 \mu \mathrm{m}$ deep trench isolation SiGe BiCMOS technology," in IEEE MTT-S Int. Microw. Symp. Dig., Fort Worth, TX, Jun. 2004, pp. 975-978.

[25] A. S. Sedra and K. C. Smith, Microelectronic Circuits, 5th ed. New York: Oxford Univ. Press, 2004, pp. 637-638.

[26] K. W. Hamed, A. P. Freundorfer, and Y. M. M. Antar, "A monolithic double-balanced direct conversion mixer with an integrated wideband passive balun," IEEE J. Solid-State Circuits, vol. 40, no. 3, pp. 622-629, Mar. 2005.

[27] S.-C. Tseng, C. C. Meng, C.-H. Chang, C.-K. Wu, and G.-W. Hung, "Broadband Gilbert micromixer with an LO Marchand balun and a TIA output buffer," in IEEE MTT-S Int. Microw. Symp. Dig., San Francisco, CA, Jun. 2006, pp. 1509-1512.

[28] D. K. Cheng, Field and Wave Electromagnetics, 2nd ed. Reading, MA: Addison-Wesley, 1989, pp. 437-444.

[29] R. Mongia, I. Bahl, and P. Bhartia, RF and Microwave Coupled-Line Circuits. Norwood, MA: Artech House, 1999, pp. 136-137.

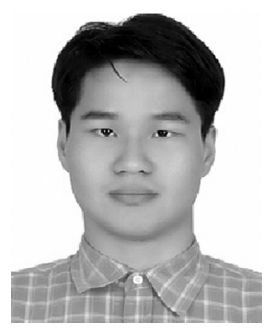

Sheng-Che Tseng (S'05) received the B.S. degree in communication engineering from National Chiao Tung University, Hsinchu, Taiwan, R.O.C., in 2003, and is currently working toward the Ph.D. degree in communication engineering from National Chiao Tung University.

His current research focuses on RF ICs and highfrequency circuitry.

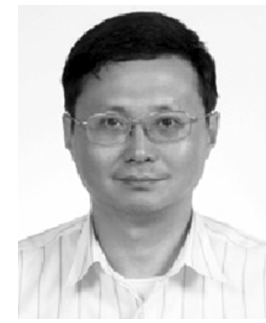

Chinchun Meng (M'02) received the B.S. degree in electrical engineering from National Taiwan University, Taipei, Taiwan, R.O.C., in 1985, and the Ph.D. degree in electrical engineering from the University of California at Los Angeles (UCLA), in 1992.

$\mathrm{He}$ demonstrated the first continuous wave $(\mathrm{CW})$ operation of a multiquantum well IMPATT oscillator at $100 \mathrm{GHz}$ during his doctoral research. He is currently an Associate Professor with the Department of Communication Engineering, National Chiao Tung University, Hsinchu, Taiwan, R.O.C. His current research interests are in the areas of RF ICs, high-frequency circuits, and highspeed devices.

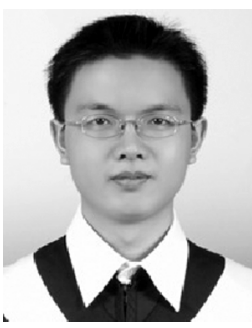

Chia-Hung Chang was born in Taipei, Taiwan, R.O.C., in 1982. He received the B.S. degree in electrical engineering from Yuan $\mathrm{Ze}$ University, Taiwan, R.O.C., in 2004, and the M.S. degree in communication engineering from National Chiao Tung University, Hsinchu, Taiwan, R.O.C., in 2006.

$\mathrm{He}$ was involved with pseudomorphic high electron-mobility transistor (pHEMT) monolithic-microwave integrated-circuit (MMIC) amplifiers and SiGe RF mixers during his master's research.

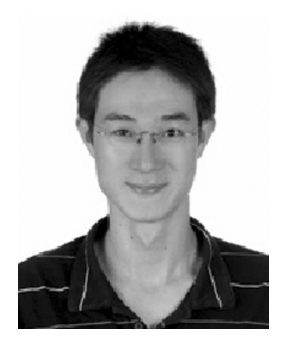

Chih-Kai Wu received the B.S. and M.S. degrees in communication engineering from National Chiao Tung University, Hsinchu, Taiwan, R.O.C., in 2003 and 2005, respectively.

From 2003 to 2005, he was with the Research Laboratory, National Chiao Tung University, where he implemented RF front-ends and 60-GHz microwave circuit design. He also focused on RF testing and on-wafer measurement with the National Nano-device Laboratory (NDL). Upon completion of the M.S. degree, he joined the Electronic Measurement Group, Agilent Technologies, Tao-Yuan, Taiwan, R.O.C., as an Application Engineer, where he supports and delivers training with wireless and mobile instruments in customer sites.

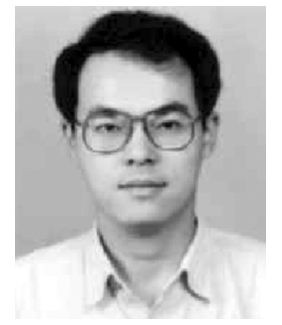

Guo-Wei Huang was born in Taipei, Taiwan, R.O.C., in 1969. He received the B.S. degree in electronics engineering and $\mathrm{Ph} . \mathrm{D}$. degree from National Chiao Tung University, Hsinchu, Taiwan, R.O.C., in 1991 and 1997, respectively.

In 1997, he joined National Nano Device Laboratories, Hsinchu, Taiwan, R.O.C., where he is currently a Researcher. His current research interests focus on microwave device design, characterization, and modeling. 